\title{
Evaluation of joint moment patterns of a wearable walking assistant robot: Experimental and simulation analyses
}

\author{
Ho Chul Kang, Ju Hwan Lee and Sung Min Kim* \\ Department of Medical Biotechnology, Dongguk University-Bio Medi Campus, 32, Dongguk-ro, \\ Ilsandong-gu, Goyang-si, Gyeonggi-do, South Korea
}

\begin{abstract}
This study analyzes the moment of human main joints (knee and hip) for developing a wearable walking assistant robot. Experiments were performed on two steps to analyze motions. Three healthy males with no neural and musculoskeletal disorders volunteered to participate in this study. In the step up test, the maximum moment was $0.98 \pm 0.05 \mathrm{Nm} / \mathrm{kg}$ for the knee and $0.52 \pm 0.04 \mathrm{Nm} / \mathrm{kg}$ for the hip. In the sit-to-stand test, the maximum moment was $0.88 \pm 0.06 \mathrm{Nm} / \mathrm{kg}$ for the knee and $0.44 \pm 0.04 \mathrm{Nm} / \mathrm{kg}$ for the hip. The moment of the hip was significantly higher than the knee. In addition, the motion analysis results were compared with proven validity and inverse dynamics analysis results. Experimental results showed that there was no significant difference in the absolute value or pattern. For the step up motion, after wearing Powered Gait Orthosis (PGO), the hip joint torque value $(1.22 \mathrm{Nm} / \mathrm{kg})$ was about 1.3 times greater than the knee joint torque value $(0.96 \mathrm{Nm} / \mathrm{kg})$. It indicates that the step up motion requires more power from the hip joint than the knee joint. Moreover, there was a significant torque value difference for before and after wearing the device.
\end{abstract}

Keywords: Simulation, Powered Gait Orthosis (PGO), torque, joint

\section{Introduction}

There are increasing numbers of patients who cannot perform independent walking because of increasing paraplegia from car and industrial accidents and disease. A number of studies for developing a walk assistance robot have been performed to solve these problems. However, the mechanical problem is that the knee joint is locked in a completely opened status for the stance and swing phases; in addition, there is increasing energy consumption and stress, so the user easily gets tired [1]. Walking speed becomes too slow [2], so it has a weakness of being less practical [1,2].

According to the findings of researchers, the frequency of using a walk assistance robot is around $25 \sim 40 \%$ in home, work and other general life situations, up until $4 \sim 5$ years after the walking training of paraplegia patients. It indicates that the results are quite lower than expectation. Therefore, it is cru-

\footnotetext{
*Address for correspondence: Sung Min Kim, Department of Medical Biotechnology, Dongguk University-Bio Medi Campus, 32, Dongguk-ro, Ilsandong-gu, Goyang-si, Gyeonggi-do, South Korea. Tel.: 82-31-961-5148; Fax: 82-31-961-5652; E-mail:smkim@dongguk.edu.
} 
cial to design a natural walk via bio-mechanical studies about various situational motions to develop a more practical walk assistance robot $[3,4]$.

Recently, as a finite element method, medical video-based body modeling technologies have been improved, so that the inverse dynamics analysis technology that predicts joint moment and muscle strength from the motion analysis information of the body musculoskeletal model has been also developed. It led biomechanical studies about the lower body that generates walking and step up motions [5]. By using inverse dynamics analysis technology, it is possible to predict the body joint moment that is generated in normal motions that cannot be measured from the experimental method via simulation. However, when measurement is performed for an actual person, it is difficult to perform a repetitive experiment since each person has a various walking style. In addition, the human body is very sensitive to external effects [6], so there is a great concern about consistency and trustworthiness. Furthermore, it is also difficult to perform exact performance measurement, because humans perform selfrewarding reactions to remove damage from the external load to a musculoskeletal system. Therefore, a simulation model development that can reduce the actual patient experimental process is a requirement.

In this study, the musculoskeletal simulation models for the sit-to-stand as well as step up motions were developed to design a practical walk assistance robot, and the inverse dynamics analysis and the motion analysis experiment were carried out. Then it is used to predict joint movements, and the moment at the pelvic limb joints for the sit-to-stand as well as the step up motions. Then it tried to study the joint load character applied to the joint area after wearing the strategic model of the wearable walk assistance robot powered gait orthosis (PGO) [7].

\section{Materials and methods}

\subsection{Motion capture}

Three examinees were selected in 20s in considerations of body mass index (BMI) to record the upper and lower body exercise (Table 1). Sit-to-stand as well as step up motions were recorded without using arms. The purpose, cautions, and experimental methods were well explained to the examinees. The balanced load is given to the left and right pelvic limbs, and they were directed to minimize upper body movements. Simple relaxing gymnastics were then performed, and reflection markers were attached to get necessary data for motion analysis.

The employed test setup for 3D motion analysis is described in Table 2. For the motion capture test, 3D motion analysis was employed and a plug-in-marker set was located for motion capture [8]. A total of 31 markers were attached on anatomical positions to record upper and lower body exercise. They also performed static pose simulation on the force plate to observe the correct positions of each joint from the display panel, as shown in Figure 1. Eight markers that caused data distortion were removed from the medial condyle during the dynamic pose.

Table 1

General anthropometric data of subjects

\begin{tabular}{|l|l|l|l|l|}
\hline & Age $(\mathrm{yr})$ & Height $(\mathrm{cm})$ & Weight $(\mathrm{kg})$ & BMI Index \\
\hline Examinee A & 25 & 181 & 74 & 22.59 \\
\hline Examinee B & 28 & 182 & 76 & 22.94 \\
\hline Examinee C & 25 & 177 & 72 & 22.98 \\
\hline
\end{tabular}


Table 2

Consist of three dimensional motion analyzer

\begin{tabular}{|l|l|l|l|l|l|l|}
\hline Kind & Force Plate & Thermo Camera & $10 \mathrm{~mm}$ Reflection marker & CCD Camera & Data processor & PC \\
\hline Number & 4 & 8 & 31 & 2 & 1 & 1 \\
\hline
\end{tabular}

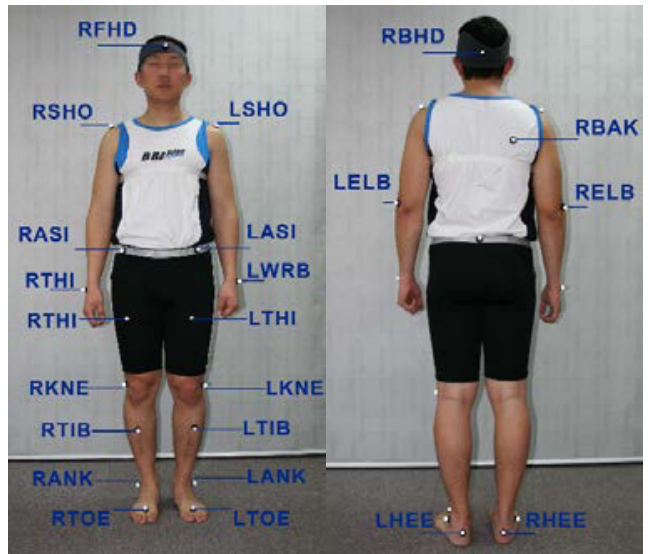

Fig. 1. Plug-in gait marker sensor attachment location of experimental model.

Two or three instances of practice were performed before starting the actual experiment. The examinee, according to the researcher's direction, was engaged in the experiment with comfortable speed, as usual. The two arms were stuck to both sides to minimize the difference between examinees due to upper body movement. The motion is then started with the researcher's sign. The space movement of
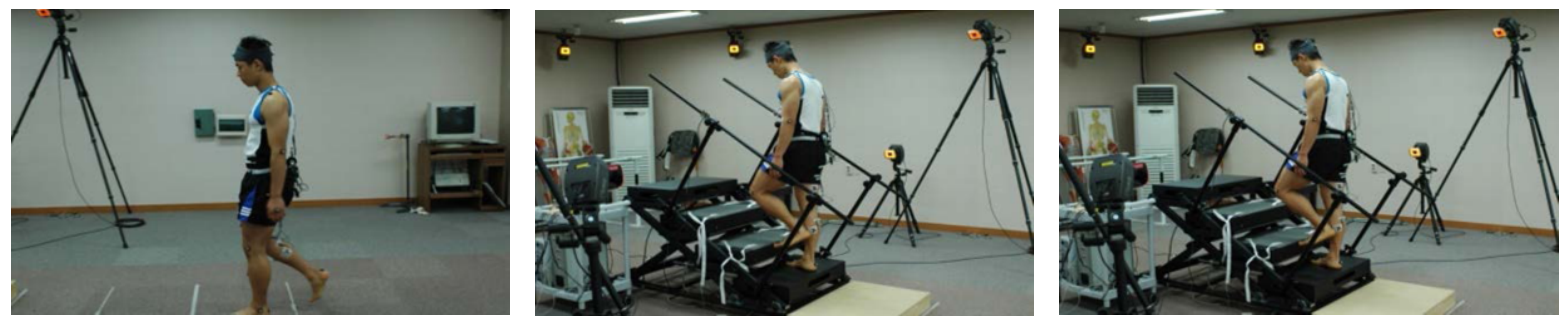

Fig. 2. Step up experimental set up \& motion capture.
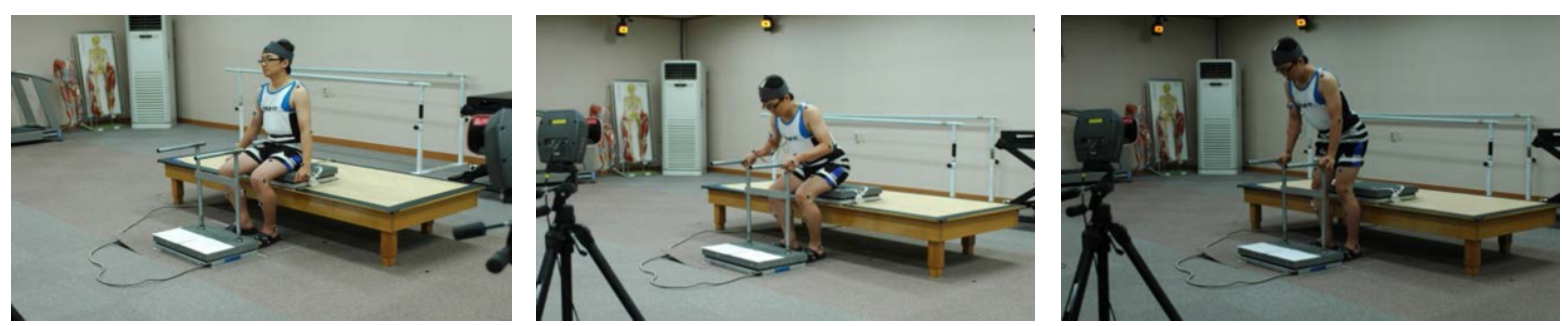

Fig. 3. Sit-to-stand experimental set up \& motion capture. 


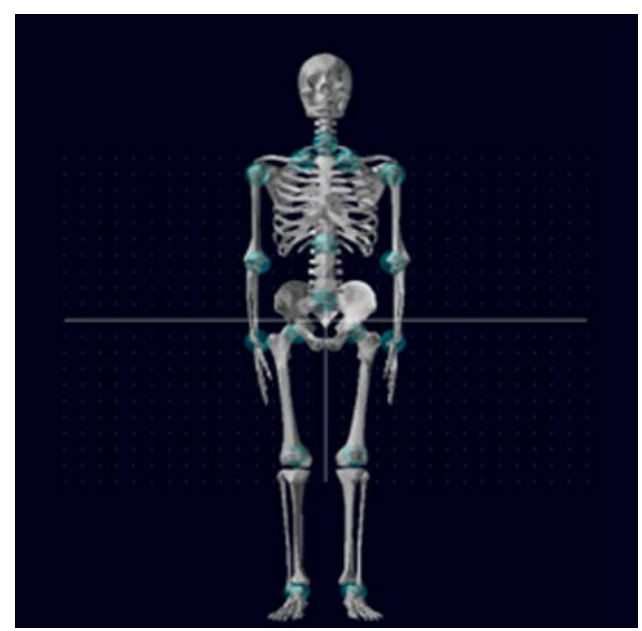

Fig. 4. Plug-in gait marker set location of simulation model.

the 23 markers during the experiment motion was measured, and the motion data values of the 23 reflection markers were used as the input value for the time processing the simulation. The input values then draw an orbit in space in each direction of $\mathrm{X}, \mathrm{Y}$, and Z, and the marker of the computer model moves according to these orbits. That is how the present method can get the same motion as actual body changes, as shown in Figures 2 and 3.

\subsection{Musculoskeletal models for upper and pelvic limbs}

This study developed a musculoskeletal model for the upper and pelvic limbs based on BRG.LifeMOD 2005.5.0 (Biomechanics Research Group, Inc., USA) to perform the inverse dynamic analysis to predict pelvic joint moment. The models were identically set as the information of the test group, and it was composed of 19 segments and 18 joints, as shown in Figure 4 [9].

\subsection{Stairs and chair modeling}

ADAMS (MSC Software corp., USA) was used to measure the parameters of the experimental chair without any arm rest and perform 3D modeling (Table 3).

\subsection{Boundary condition}

The examinees adjust many joints to make more a natural pose, but the virtual model has fewer joints to control, so mostly it provides unnatural poses, but the basic structure position or angle is almost identical to the actual examinee, as shown in Figure 5.

Figure 6 presents the integration of the reflection markers shown in Figure 1 and the motion agents. The constraint between the body model and ground is set to enable walking on the surface with contact of the sole and stair, as shown in Figure 7. The constraint between the body model and the stair is set so that the body model's sole contacts the surface of the stair, and the body model does not fall down the stairs when gravity is given, as shown in Figure 8. The constraint of the body model and the chair is set to contact the chair, so that the body model would not fall when the gravity is given (Figure 9). 
Table 3

Stair and chair size for 3D modeling

\begin{tabular}{|l|l|l|l|l|}
\hline & Length $(\mathrm{mm})$ & Height $(\mathrm{mm})$ & Width $(\mathrm{mm})$ & Characteristic \\
\hline Stair Size & 770 & 100 & 276 & $\begin{array}{l}\text { Without Handrail } \\
\text { 4STEP }\end{array}$ \\
\hline Chair Size & 600 & 500 & 395 & Without Handrail \\
\hline
\end{tabular}
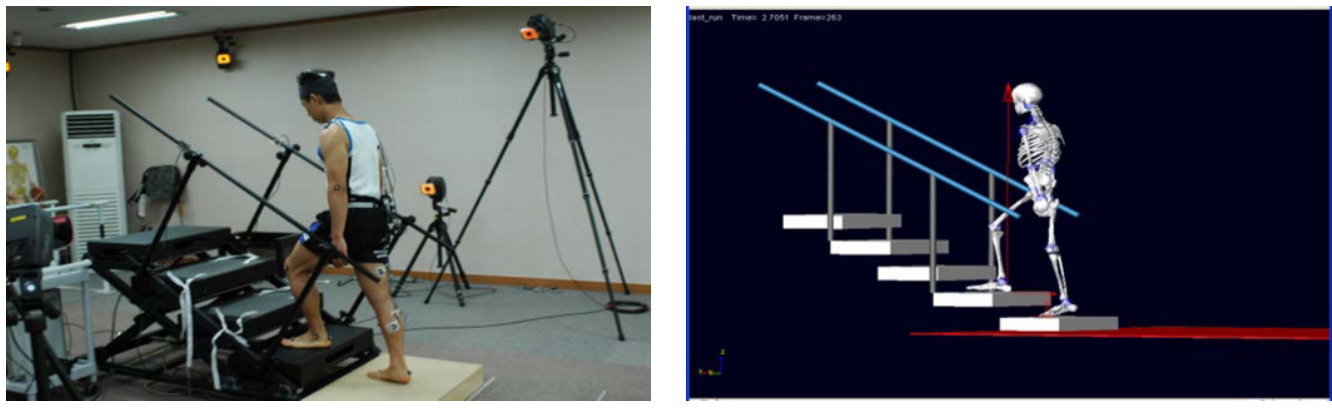

(a) Modeling of step up position
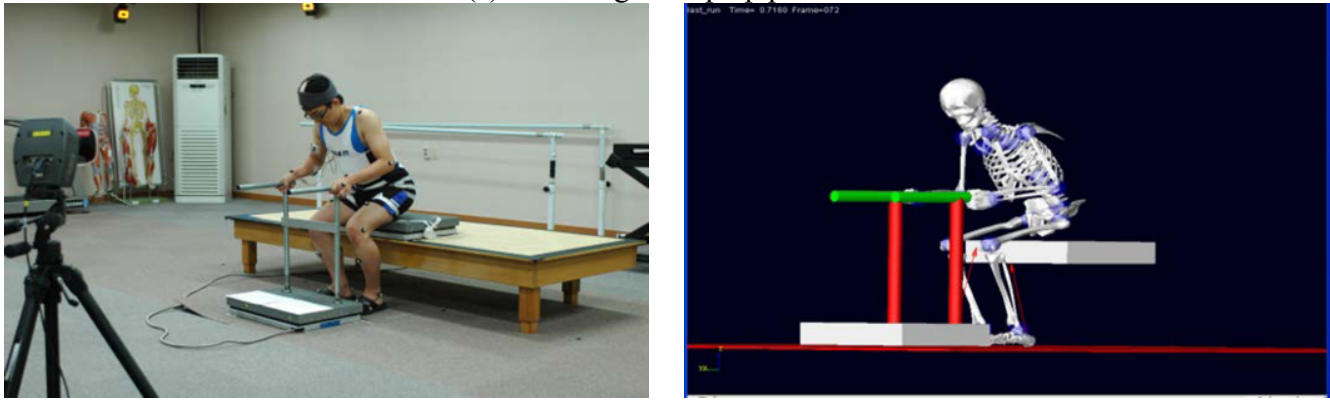

(b) Modeling of a sit-to-stand position

Fig. 5. Experimental model and simulation model.

The PGO model developed by each situational body model and rehabilitation engineering research lab was brought for each part and constrained via bushing (the connection factor with 12 characters). The property for each part was applied by aluminum, and a motor with a predicted weight of $1 \mathrm{~kg}$ each was installed on the pelvic limbs. The predicted weight of the back pack was $5 \mathrm{~kg}$. Then, the link system was released to allow free movement of the joint part during the simulation, as shown in Figure 10.

\section{Results}

The joint motion information and joint moment results in the motion of step up and sit-to-stand were compared to the experimental results to prove the suitability of joint motion information measured from the simulation experiment of the research. The joint moment was divided by body weight to offset the effect of the examinee's body weight on joint moment. The joint moment predicted from inverse dynamics analysis was compared with the motion analysis result and proved the validity of the developed body model program. 


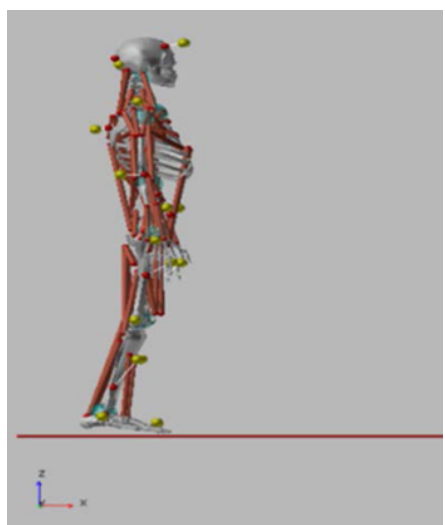

Fig. 6. Reflective marker set and motion agents.

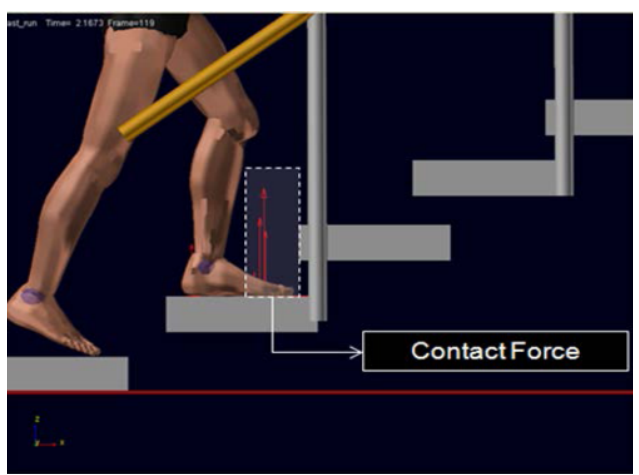

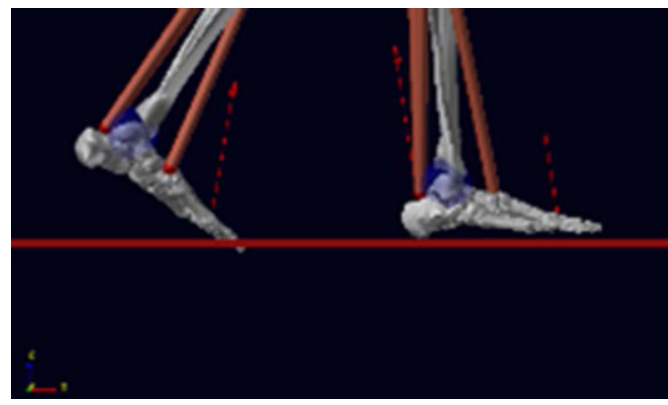

Fig. 7. Contact force between a surface and human body model.

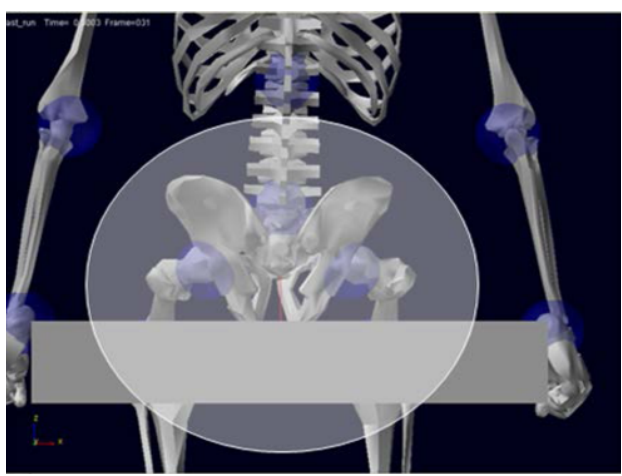

Fig. 8. Contact force between stairs and human body models. Fig. 9. Contact force between chair and human body model.

\subsection{Human model parameter acquisition and verification}

The normalized joint moment result of the step up and sit-to-stand motions with the body weight of the three examinees calculated from motion analysis is shown in Table 4. The joint moment of the hip and knee all showed a small value at the start and both continuously increased the joint moment for the forward speeding step. Its maximum was $0.98 \mathrm{Nm} / \mathrm{kg}, 0.88 \mathrm{Nm} / \mathrm{kg}$ and $0.52 \mathrm{Nm} / \mathrm{kg}, 0.44 \mathrm{Nm} / \mathrm{kg}$ for the knee joint in the step up and standing up motion.
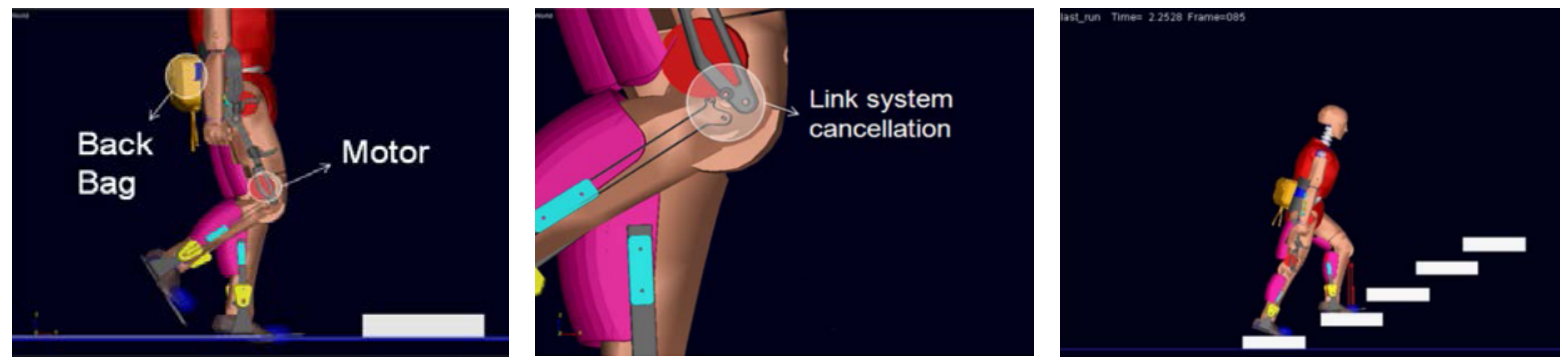

Fig. 10. Wearable PGO models. 
Table 4

Stair up and sit-to-stand results

\begin{tabular}{|l|l|l|l|}
\hline \multicolumn{2}{|c|}{} & Mean $\mathrm{M}_{\text {hip }}$ maximum $(\mathrm{Nm} / \mathrm{kg})$ & Mean $\mathrm{M}_{\text {knee }}$ maximum $(\mathrm{Nm} / \mathrm{kg})$ \\
\hline \multirow{3}{*}{ Stair up } & Motion Analysis & $0.98(0.05)$ & $0.52(0.04)$ \\
\cline { 2 - 4 } & Simulation & $0.97(0.07)$ & $0.61(0.04)$ \\
\hline \multirow{3}{*}{ STS } & Motion Analysis & $0.88(0.06)$ & $0.44(0.04)$ \\
\cline { 2 - 4 } & Simulation & $0.83(0.05)$ & $0.43(0.05)$ \\
\hline
\end{tabular}

The normalized hip and knee joint moment showed a similar absolute value or pattern other than the different maximum value generation time in comparison with the previous result [10-12]. The difference of generation time of the maximum joint moment occurs mainly due to different patterns of ankle joint for each examine, which delays the generation of the hip and knee joint moments.

The motion analysis results were compared and analyzed with proven validity and inverse dynamics analysis results. Comparison and analysis for the three examinees in each test group showed that there was no significant difference in the absolute value or pattern (Table 4, Figures 11 and 12). Moreover, the upper and lower limb models were developed by comparing the joint motion information predicted from inverse dynamics analysis and the motion experiment.

\subsection{Wearable PGO step up}

The changed hip and knee joint moment values were derived after wearing PGO via computed virtual analysis by ADAMS (MSC Software corp., USA) while climbing stairs with the developed upper and pelvic limb modeling and the stair model. While going up the stairs, the maximum torque value at the mid-sagittal plane of the knee joint was larger than that of the hip joint. In addition, the walking speed was reduced than regular walking when wearing PGO, while the maximum torque vale increased for both, as shown in Figure 13.

\section{Conclusions}

This study derived design variables for the purpose of optimum design for wearable walk assistance robots. Regular walk of 20 s adult, step up motions, and sit-to-stand motions were used to develop the body model. The developed model can predict the major pelvic moment that is generated from the step up motion after wearing PGO in the appropriate range, which was not measured in previous experimental methods. The following conclusions were derived from the experimental results according to the step up motion after wearing PGO.

For the step up motion after wearing PGO, the hip joint torque value $(1.22 \mathrm{Nm} / \mathrm{kg})$ was about 1.3 times greater than the knee joint torque value $(0.96 \mathrm{Nm} / \mathrm{kg})$, which means that the step up motion requires more power from the hip joint than the knee joint. Also, there was a significant torque value difference for before and after wearing the device. Standardized chair and stair models that can describe more complex motion of the joint and measure of more accurate movements of each pelvic joint are required. The wearable walk assistance robot receives different power for each user. Therefore, additional study about pelvic joints according to body motion change in various situations is needed to develop more a practical walk assistance robot. Also, there must be studies with a greater number of 


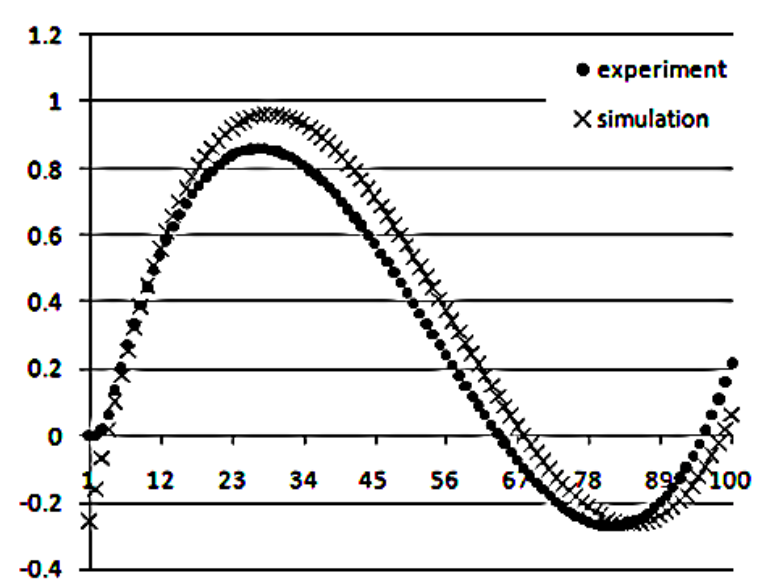

(a) Hip moment of examinee A

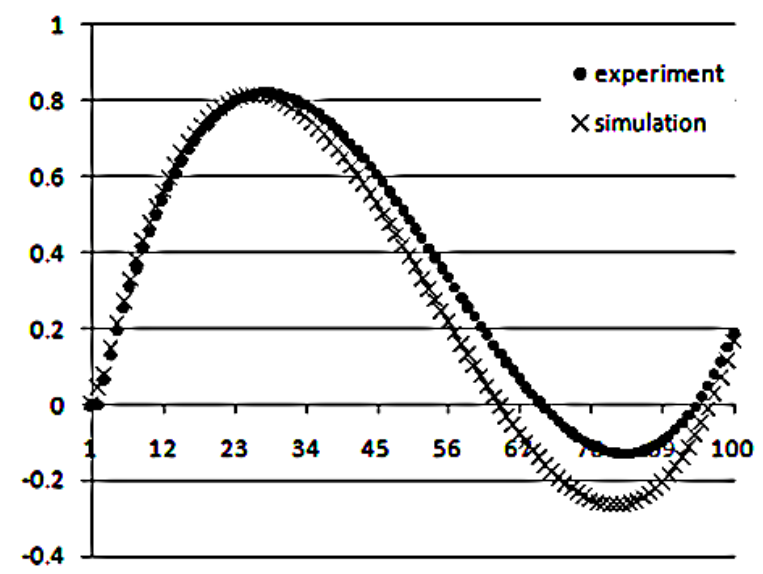

(c) Hip moment of examinee B

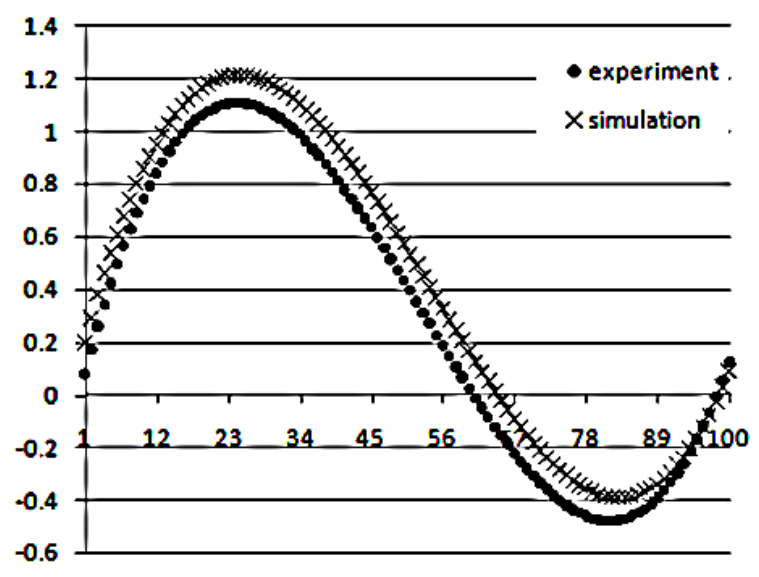

(e) Hip moment of examinee C

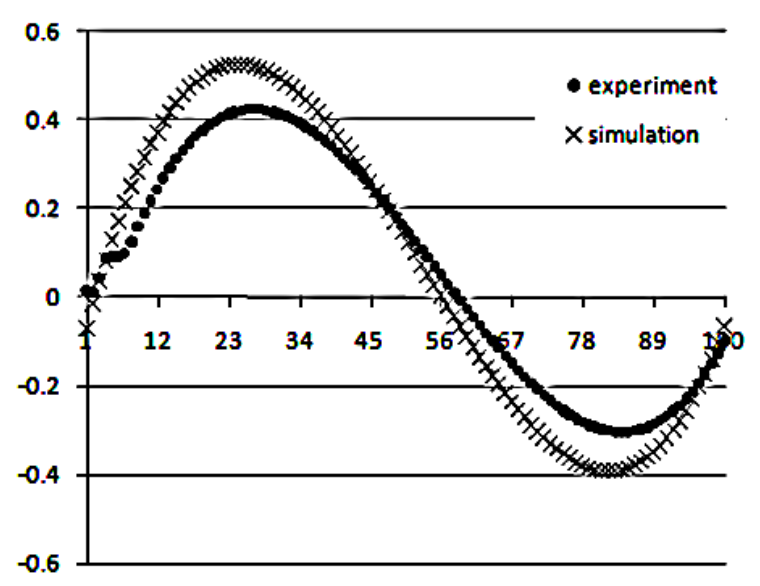

(b) Knee moment of examinee A

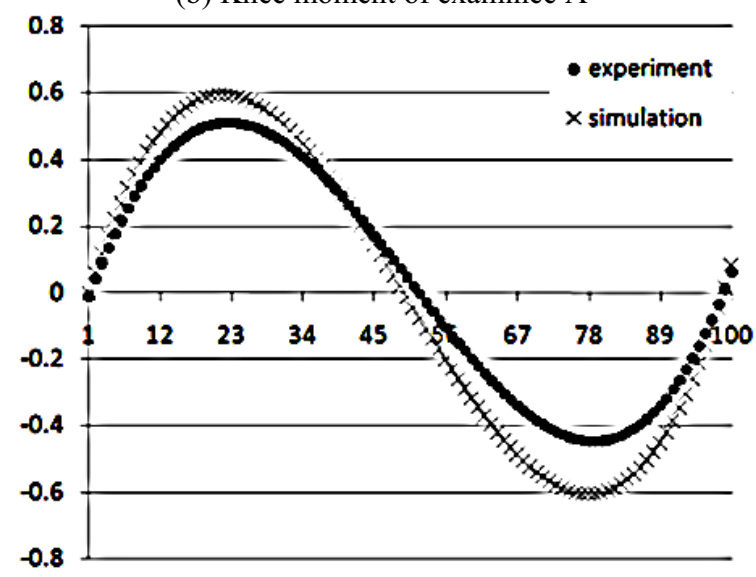

(d) Knee moment of examinee B

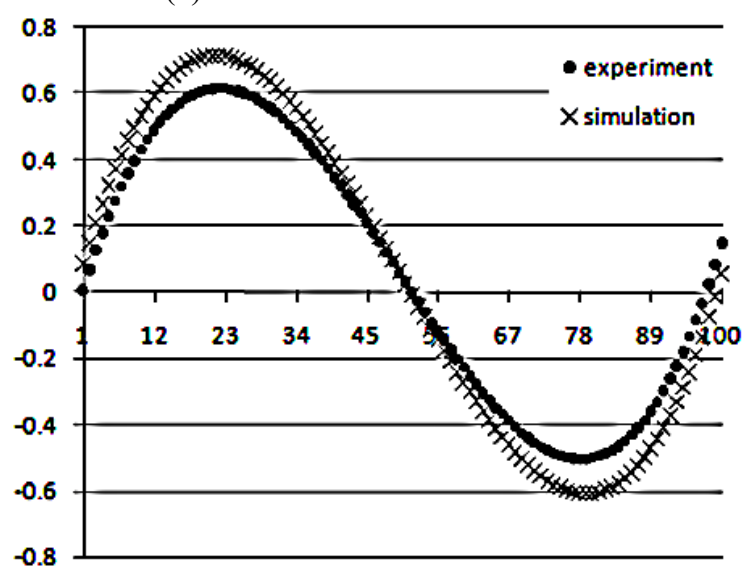

(f) Knee moment of examinee $\mathrm{C}$

Fig. 11. Comparison of step up results between experiment and simulation. 


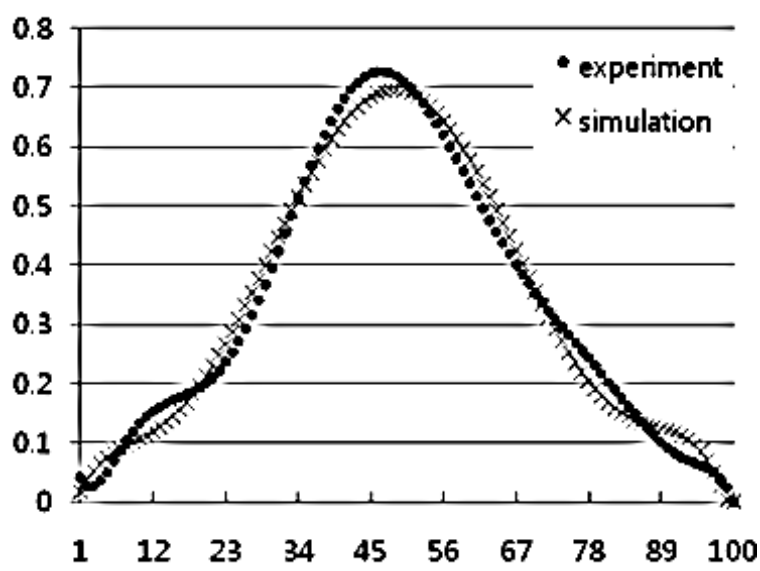

(a) Hip moment of examinee A

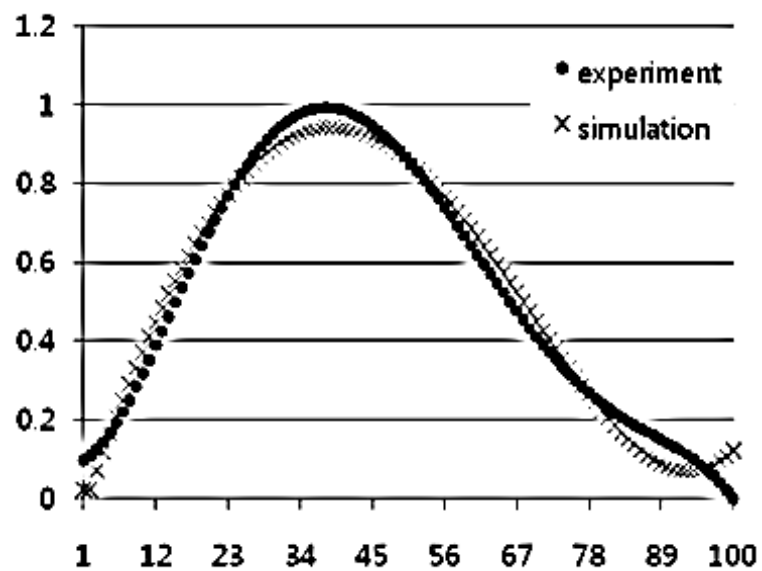

(c) Hip moment of examinee B

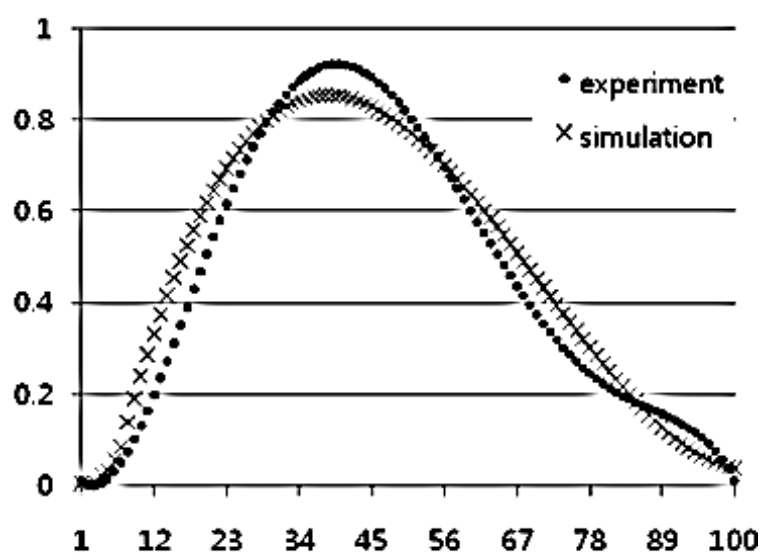

(e) Hip moment of examinee $\mathrm{C}$

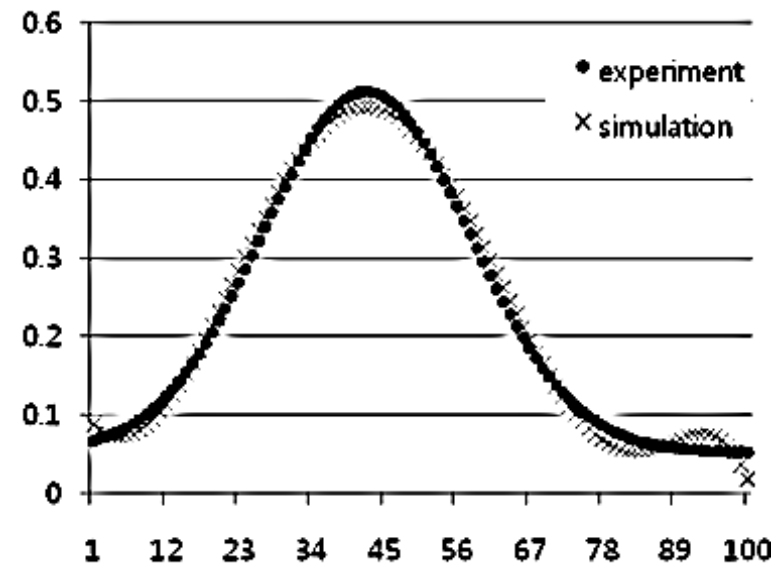

(b) Knee moment of examinee A

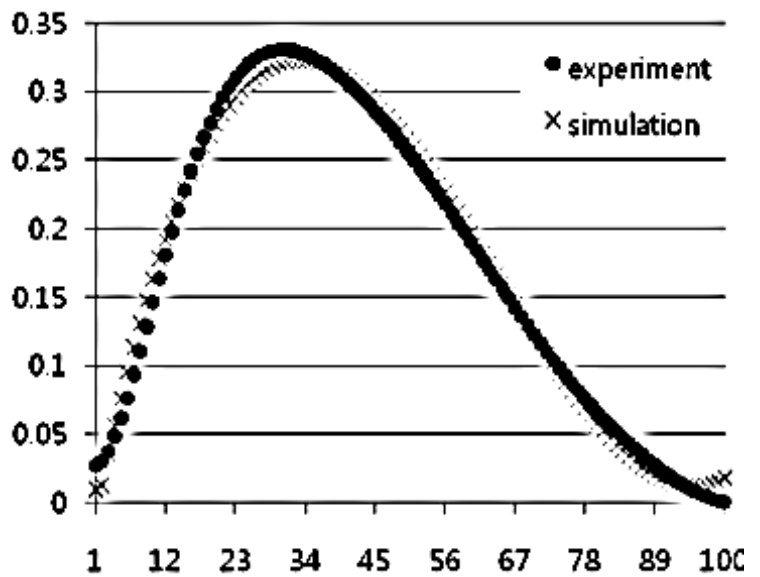

(d) Knee moment of examinee B

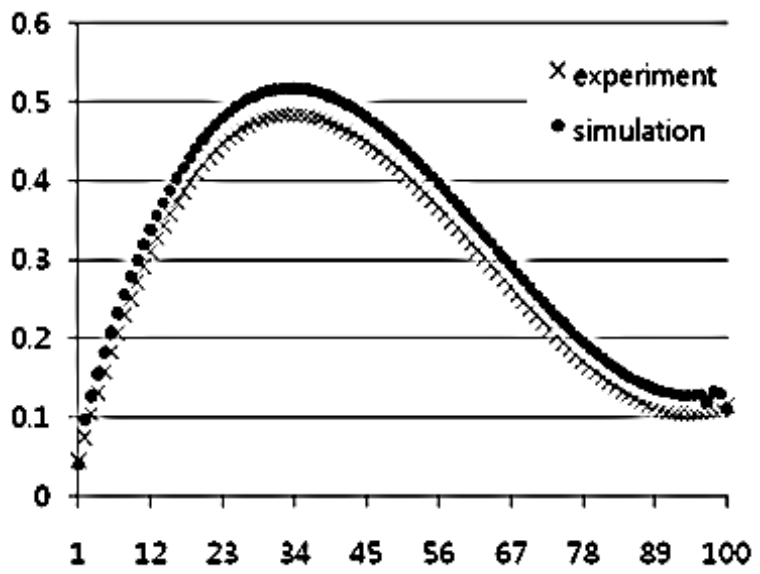

(f) Knee moment of examinee $\mathrm{C}$

Fig. 12. Comparison of sit-to-stand results between experiment and simulation. 


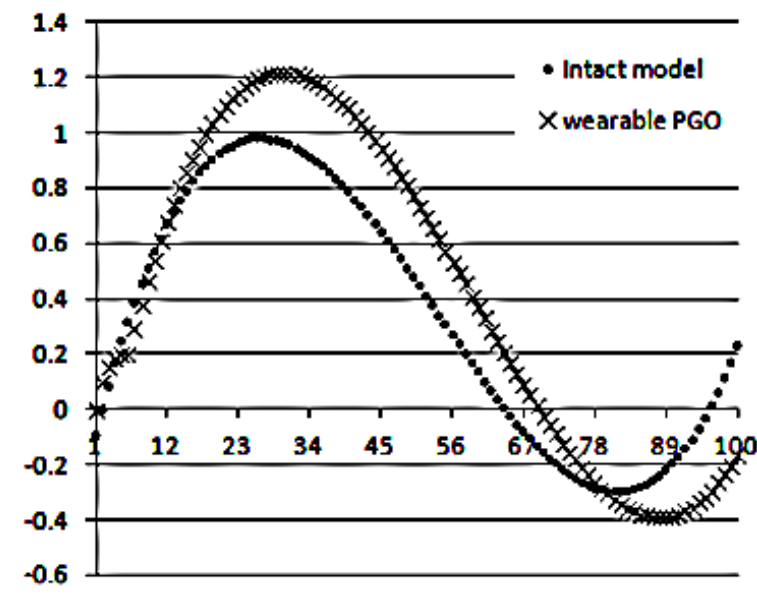

(a) Hip moment

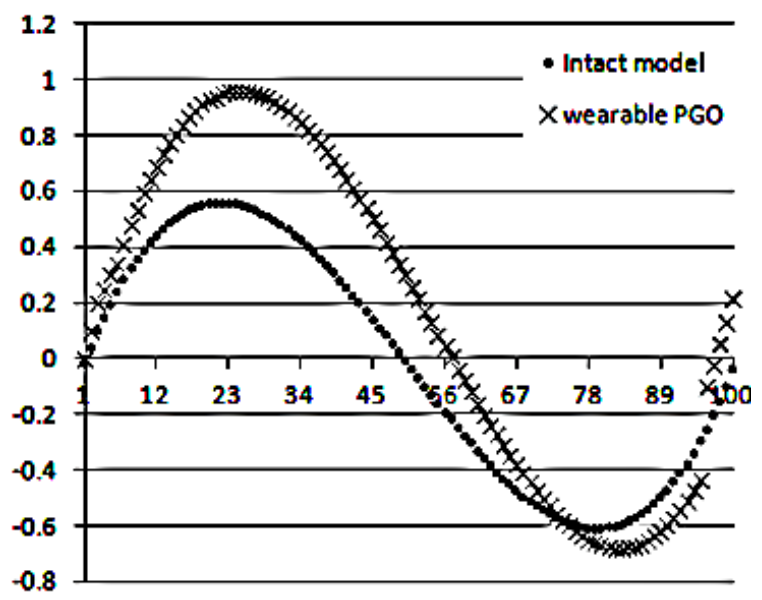

(b) Knee moment

Fig. 13. Comparisons of step up results between the intact model and wearable PGO model from simulation.

examinees along with consistent study of normal adults prior to application to disabled people, since it is only the early stage of development.

In addition, the result analysis process only referred to the biodynamic properties of pelvic joints, since the study only used stairs without a handrail and a chair; reference data of motion using handrails must be included for comparison and analysis. In conclusion, for the stair walk using the walk assistance robot, consideration must be given to each joint part to decide the specifications of the joint motor of the wearable walk assistance robot.

\section{Acknowledgment}

This study was supported by a grant of the Korea Healthcare Technology R\&D Project, Ministry of Health \& Welfare, Republic of Korea (A102058).

\section{References}

[1] B. Graf, M. Hans and R.D. Schraft, Care-O-bot II--development of a next generation robotic home assistant, Autonomous Robots 16 (2004), 193-205.

[2] E.M. Coletta and J.B. Murphy, The complications of immobility in the elderly stroke patient, The Journal of the American Board of Family Practice 5 (1992), 389-397.

[3] W.B. Johnson, S. Fatone and S.A. Gard, Walking mechanics of persons who use reciprocating gait orthoses, Journal of Rehabilitation Research and Development 46 (2009), 435-446.

[4] B.J. Ruthenberg, N.A. Wasylewski and J.E. Beard, An experimental device for investigating the force and power requirements of a powered gait orthosis, Journal of Rehabilitation Research and Development 34 (1997), 203-213.

[5] S. Sathasivam and P.S. Walker, Computer model to predict subsurface damage in tibial inserts of total knees, Journal of Orthopaedic Research 16.5 (1998), 564-571.

[6] M. Griffin, Handbook of Human Vibration, Academic Press, London, 1990.

[7] S. Kang, J. Ryu, G. Kim and M. Mun, Hip joint control of PGO for paraplegics, Key Engineering Materials 326-328 (2006), 735-738.

[8] J. Perry, Gait analysis, Rancho Los Amigos Medical Center, Downey, CA. SLACK Incorporated, 2010.

[9] T. Maeda and S. Tachi, Human system learning model for solving the inverse kinematics problem by direct inverse modeling, Systems and Computers in Japan 27 (1996), 53-68. 
[10] A. Stacoff, C. Diezi, G. Luder, E. Stüssi and I.A. Kramers-de Quervain, Ground reaction forces on stairs: Effects of stair inclination and age, Gait \& Posture 21 (2005), 24-38.

[11] J.E. Zachazewski, P.O. Riley and D.E. Krebs, Biomechanical analysis of body mass transfer during stair ascent and descent of healthy subjects, Journal of Rehabilitation Research and Development 30 (1993), 412-422.

[12] W.G. Janssen, H.B. Bussmann and H.J. Stam, Determinants of the sit-to-stand movement: A review, Physical Therapy 82 (2002), 866-879. 